\title{
Production orale de l'accent lexical espagnol par les francophones : une étude expérimentale
}

The production of lexical stress by French learners of Spanish: an experimental study

\section{Margarita Muñoz Garcia}

\section{OpenEdition}

Journals

Édition électronique

URL : http://journals.openedition.org/tipa/1242

DOI : $10.4000 /$ tipa. 1242

ISSN : 2264-7082

Éditeur

Laboratoire Parole et Langage

Référence électronique

Margarita Muñoz Garcia, «Production orale de l'accent lexical espagnol par les francophones : une étude expérimentale », TIPA. Travaux interdisciplinaires sur la parole et le langage [En ligne], 30 | 2014, mis en ligne le 05 janvier 2015, consulté le 19 avril 2019. URL : http://journals.openedition.org/ tipa/1242 ; DOl : 10.4000/tipa.1242

Ce document a été généré automatiquement le 19 avril 2019.

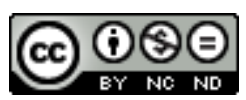

La revue TIPA. Travaux interdisciplinaires sur la parole et le langage est mise à disposition selon les termes de la licence Creative Commons Attribution - Pas d'Utilisation Commerciale - Pas de Modification 4.0 International. 


\title{
Production orale de l'accent lexical espagnol par les francophones: une étude expérimentale
}

The production of lexical stress by French learners of Spanish: an experimental study

\author{
Margarita Muñoz Garcia
}

\section{Introduction}

1 Ce travail s'inscrit dans le domaine de l'apprentissage des langues étrangères. Le point de départ de la présente étude est notre pratique d'enseignement de l'espagnol langue étrangère (ELE). Au niveau phonétique, l'étude de l'interlangue ${ }^{1}$ suscite de nombreuses recherches, tant au niveau segmental que suprasegmental. Les éléments suprasegmentaux jouent un rôle essentiel en raison de la fonction primordiale qu'ils ont dans le processus d'intégration du message (Cortés, 2002: 71). En conséquence, un approfondissement de l'étude de la prosodie de l'interlangue peut contribuer à mieux comprendre le processus d'acquisition de la prononciation, plus particulièrement de la prosodie, et à mieux se confronter à l'enseignement et l'apprentissage de la prononciation d'une langue étrangère (L2).

2 Nous nous intéressons à l'apprentissage de la prosodie par des francophones, notamment en ce qui concerne la production de l'accent lexical espagnol. Selon Rossi (1999: 7) :

« (...) la prosodie est un ensemble constitué de l'accentuation, de l'intonation et du rythme (...) L'accentuation et l'intonation sont des structures prosodiques pluridimensionnelles (on dit pluriparamétriques) définies par un ensemble de traits. L'accentuation et l'intonation se manifestent dans un espace prosodique à trois dimensions. Ces dimensions objectives sont la fréquence fondamentale, l'intensité et le temps. » 
3 L'accent est le trait prosodique qui permet de mettre en relief une unité linguistique supérieure au phonème (syllabe, morphème, mot, syntagme ou phrase) et qui fonctionne comme une unité du niveau supérieur pour la différencier des autres unités linguistiques du même niveau. De ce fait, l'accent se manifeste comme un contraste entre unités accentuées et inaccentuées (Quilis, 1993 : 388). Dans ce contexte, il existe une différence d'accentuation entre les deux langues concernant cette étude. Le français possède un accent primaire fixe oxyton : il frappe toujours la dernière syllabe du mot, du syntagme ou de la phrase. La langue française se caractérise par un accent de groupe et non un accent de mot. Lorsque les mots sont rassemblés de façon à former une unité plus grande (un groupe de mots, une phrase), ils sont accentués ou non en fonction de la place qu'ils occupent dans le groupe accentuel (Di Cristo, 1981: 32). Sur le plan linguistique, cet accent possède une fonction démarcative qui facilite le décodage des unités de sens ou syntagmes (Léon, 1992: 109). En revanche, l'espagnol est une langue à accent partiellement libre, l'accent peut survenir sur l'une des quatre dernières syllabes du mot. Dans un mot, par exemple, de trois syllabes, l'accent peut frapper sur la première, la deuxième ou la troisième syllabe ('límite, li'mite, limi'tée). L'accent espagnol possède une fonction distinctive qui agit sur l'axe paradigmatique. Le changement de place de cet accent de la syllabe tonique sert à distinguer deux unités de signification différentes (Quilis, 1993 : 388-390). En fonction de l'endroit où se trouve l'accent dans les mots, on peut différencier en espagnol quatre types de accentuation : a) mots oxytons : la syllabe accentuée est la dernière du mot (situa'ción ou car'tel); b) mots paroxytons : la syllabe accentuée est l'avant-dernière du mot (co'media, 'pata) ; c) mots proparoxytons : la syllabe accentuée est l'antépénultième du mot ('mágica ou 'pájaro) et d) mots superproparoxytons (formes verbales suivies d'un ou plusieurs pronoms enclitiques) : la syllabe accentuée est celle avant l'antépénultième du mot (presen'tándomelo, co'giéndosela).

4 Nous devons préciser que l'espagnol possède des règles d'orthographie précises qui marquent la position de l'accent du mot par un accent écrit. Par exemple, lorsque le mot finit par une voyelle un $n$ ou un $s$ et qu'il n'y a pas d'accent écrit, le mot sera obligatoirement paroxyton; les mots proparoxytons ont toujours un accent écrit; si le mot finit par une 'voyelle' un 'n' ou un 's' et il est accentué sur la dernière syllabe, ce mot oxyton est également accentué à l'écrit, etc. L'influence de l'orthographe dans la production des items dépasse les cadres de cette étude. Comme nous précisons plus tard lorsque nous exposons la procédure de passation du test en 3.3., nous avons présenté les stimuli de manière délibérée à l'aide d'images et non le mot écrit ${ }^{3}$.

5 Par ailleurs, l'influence de la langue maternelle (LM) sur les productions en L2 est un fait constaté tant au niveau segmental que suprasegmental (Wieden, 1993: 130-131; Archibald, 1997: 277-278, in Rasier, 2003: 81; Cortés, 2002: 53; Rasier \& Hiligsmann, 2007 : 59). La littérature fait écho à la tendance des francophones à placer l'accent sur la dernière syllabe du mot (Sarmiento et al., 1974 : 51-75; Fernández González, 1998: 133; Iruela, 2004 : 41; Gil, $2007: 288$ ). Le but principal de cette étude est de vérifier l'influence de la LM sur le traitement de l'accent lexical espagnol par des francophones lors de la production orale, en tenant compte des caractéristiques des deux langues et de leurs différences d'accentuation. Le travail qui suit tente ainsi de répondre à ces questions et de tester chacune des hypothèses qui y sont associées à la lumière des résultats des tests de production orale mis en place. 


\section{Hypothèses}

6 Afin de mieux comprendre l'élaboration des hypothèses émises et du protocole expérimental, nous tenons à préciser que ceci est un extrait d'une expérimentation plus large qui a fait l'objet d'une thèse qui étudie la perception et la production de l'accent lexical espagnol par des apprenants francophones (Muñoz Garcia, 2010). Dans cette thèse, nous avons comparé comment les francophones perçoivent et produisent l'accent espagnol en observant les mêmes stimuli lors de deux tâches (perception et production). En outre, nous avons analysé les réalisations acoustiques des locuteurs francophones et les avons comparées à celles de locuteurs hispanophones (nous avons observé trois facteurs : fréquence fondamentale, durée et niveau de registre tonal). En conséquence, une partie des hypothèses qui suivent découlent parfois de constatations faites lors des études précédentes (Muñoz Garcia et al., 2009, et Muñoz Garcia, 2010) qui ne concernent que la perception afin de vérifier si nous observons les mêmes effets lors de la production orale de l'accent lexical.

\subsection{Hypothèse générale : effet de la LM}

7 L'un des objectifs de ce travail est de tester l'incidence de la langue maternelle sur le nombre de bonnes réponses (BR) dans la production de l'accent en ELE. Dans le cadre de l'étude que nous avons menée on considère une BR lorsque l'accent est perçu par l'évaluateur sur la syllabe qui correspond à la syllabe accentuée du mot. Par exemple, dans le mot oxyton pa'red, l'évaluateur perçoit l'accent sur la dernière syllabe du mot 'red.

Pour établir notre hypothèse, nous prenons en considération les différents constats recensés dans la littérature faisant référence à l'influence de la LM sur les productions en L2 ( $c f .1)$, et plus particulièrement à la pratique récurrente des francophones de placer l'accent sur la dernière syllabe. En outre, dans notre pratique d'enseignante d'ELE à des francophones, nous observons des productions considérablement influencées par leur LM au niveau suprasegmental, se traduisant par une tendance à déplacer l'accent systématiquement sur la dernière syllabe. En conséquence, nous présumons que les performances des sujets francophones sont certainement influencées par leur LM et présentent un déplacement de l'accent sur la dernière syllabe.

\subsection{Hypothèses particulières}

\subsubsection{Effet du 'niveau des sujets'}

9 Au niveau de la production, des travaux sur l'observation d'apprenants néerlandais du français montrent que l'on peut identifier plusieurs stades successifs dans l'évolution de leur interlangue, où celle-ci se restructure peu à peu (Rasier, 2003 : 83). Par ailleurs, des travaux menés sur la production de l'accent lexical espagnol par des apprenants francophones montrent que « plus le niveau d'apprentissage est élevé, plus ils produisent correctement l'accent espagnol » (Lafrance, 2001 : 111). Nous partons de l'hypothèse que plus le niveau de langue est élevé, moins les erreurs se produiront. En d'autres termes, au cours de l'apprentissage de la L2 (en l'occurrence de l'ELE), nous postulons que les apprenants acquièrent au fur et à mesure une certaine maîtrise de l'accentuation, grâce à laquelle les erreurs en production auront tendance à disparaitre. 


\subsubsection{Effet des 'mots isolés ou mots en contexte'}

10 L'étude porte sur l'observation de la production de l'accent des mots qui sont présentés isolés et de ces mêmes mots insérés dans une phrase en position finale. Par exemple, le mot cama est présenté de façon isolée mais aussi inséré dans une phrase assertive « Pablo duerme en su cama ", et dans une phrase interrogative "¿Pablo duerme en su cama?".

11 Nous fondant sur notre expérience d'enseignement, nous postulons que la production de l'accent lexical est plus difficile en contexte (mot-cible inséré dans une phrase) que dans les mots présentés isolément. En effet, l'augmentation des mots à traiter rend la tâche cognitive plus compliquée. Par exemple, l'accent lexical du mot "'gato » sera plus difficile à prononcer correctement lorsqu'il est inséré dans la phrase « No te gusta este 'gato » que lorsqu'il est présenté isolément.

\subsubsection{Effet du 'type d'intonation'}

12 Les résultats d'autres études précédentes ont révélé une difficulté particulière des francophones à identifier l'accent lexical lors des expériences de perception auditive dans le contexte de phrases à intonation montante (Muñoz García et al., 2009: 494 et Muñoz García, 2010 : 163-164). Nous nous demandons si l'intonation montante ou descendante joue également un rôle lors de la production de l'accent lexical et nous postulons que l'intonation montante peut rendre la tâche de production plus difficile. Ceci confirmerait les résultats obtenus dans notre expérience de perception citée ci-dessus.

\subsubsection{Effet du 'nombre de syllabes'}

13 En fonction du nombre de syllabes des mots, la production peut s'avérer plus difficile. Nous supposons que plus le mot contient de syllabes, plus la production de l'accent lexical peut s'avérer difficile. Les résultats de Lafrance (2001: 112-113) lors d'une étude de production de parole d'étudiants francophones vont aussi dans ce sens.

\subsubsection{Effet du 'type d'accentuation'}

14 Nous avançons deux hypothèses sur la question du type d'accentuation. La première concerne l'exposition aux différents types d'accentuation. Premièrement, nous nous demandons s'il existe des degrés de difficulté différents en fonction du 'type d'accentuation'. En raison de la fréquence globale dans la langue, il est permis de présumer que les sujets obtiennent les meilleures performances pour les mots paroxytons, puisque approximativement $80 \%$ des mots espagnols sont paroxytons. Une exposition plus fréquente à l'accentuation paroxytone peut donc influencer la production de l'apprenant francophone.

15 La deuxième hypothèse concerne la similarité entre les deux langues. En effet, lors de la précédente étude sur la perception de l'accent lexical (Muñoz Garcia, 2010 : 166) nous avons observé que l'accentuation la plus difficile à identifier par les sujets francophones concernait les mots oxytons de trois syllabes. De ce fait, nous pensons que la similarité du type d'accentuation peut poser également davantage de difficultés lors de la production. Par conséquent, nous présumons que les mots oxytons seront plus difficiles à prononcer 
que les deux autres types d'accentuation. Les sujets auront plus de difficulté à produire, par exemple, l'accent du mot « Pa'rís» que du mot « mu'jer».

\subsubsection{Effet de la 'structure accentuelle'}

16 Le facteur 'structure accentuelle' est une combinaison des facteurs 'type d'accentuation' et 'nombre de syllabes'. Cet effet est pris en compte afin d'examiner si les hypothèses concernant l'un et l'autre ont des impacts différents lors de la combinaison de ces deux facteurs. Pour chaque structure accentuelle, nous avons sélectionné des mots de deux et de trois syllabes, à l'exception des mots proparoxytons, qui ne peuvent être que trisyllabiques. L'échantillon de stimuli comporte donc 5 types de structure accentuelle :
a) 8 mots de 2 syllabes oxytons : ex. a'zul, ma'rrón
b) 8 mots de 2 syllabes paroxytons : ex. 'mesa, 'taxi
c) 8 mots de 3 syllabes oxytons : ex. cora'zón, hospi'tal
d) 8 mots de 3 syllabes paroxytons : ex. ca'beza, gui'tarra,
e) 8 mots de 3 syllabes proparoxytons : ex. 'sábado, 'música

\subsubsection{Effet de la 'transparence lexicale'}

De la même manière que l'on présume que la similarité peut poser des problèmes dans le cas d'une accentuation similaire (oxytone) entre les deux langues, nous avons voulu observer si l'effet de la similarité phonétique entre les mots des deux langues peut s'avérer significatif. Ainsi, nous examinons si la ressemblance segmentale phonétique entre les mots français et espagnols peut être une source de difficulté. Notre hypothèse prévoit que la production de l'accent lexical sera plus difficile pour les «mots transparents» (mots proches phonétiquement en français et en espagnol) que pour les mots «non transparents» (pas de ressemblance phonétique entre les deux mots). Les mots transparents sélectionnés pour l'expérience peuvent différer ou non dans l'accentuation du mot dans les deux langues. Par exemple : «me'lón / melon », «'taxi / taxi ", "'número / le numéro et nu'méro / je numérote ». Les mots non transparents sont des mots qui n'ont aucune ressemblance phonétique entre les deux langues. Par exemple : « a'zul / bleu », « re'loj / montre », «'pájaro / oiseau ».

\section{Procédure expérimentale}

Le test expérimental a été élaboré sur la base de l'ensemble des hypothèses formulées supra.

\subsection{Sujets}

19 L'échantillon de cette expérience est composé de vingt-quatre sujets francophones et d'un groupe contrôle de huit sujets hispanophones (la moyenne d'âge est de 23 ans, âges compris entre 19 et 40 ans). Afin de vérifier l'hypothèse concernant l'effet du 'niveau des sujets' nous avons choisi des étudiantes universitaires ${ }^{5}$ avec des niveaux de langue différents correspondant à un des trois niveaux de langue décrits par le Cadre Européen Commun de Référence pour l'Apprentissage / Enseignement de Langues : a) 8 sujets du niveau $A$, appelés utilisateurs élémentaires ${ }^{6} ;$ b) 8 sujets du niveau $B$, appelés utilisateurs indépendants ${ }^{7}$; c) sujets du niveau $C$, appelés utilisateurs expérimentés ${ }^{8}$. 
les sujets francophones sont d'origine française et obligatoirement monolingues de langue maternelle française'. L'échantillon de sujets de LM espagnole comprend des personnes de niveau universitaire, soit des étudiants universitaires au moment de l'expérience, soit des personnes ayant déjà fini leurs études (cinq ans universitaires). Tous ces locuteurs possèdent une prononciation standard de l'espagnol et ne présentent aucune pathologie du langage.

\subsection{Stimuli}

En fonction des variables indépendantes de l'étude, nous avons sélectionné 40 stimul consistant en des mots très courants ${ }^{10}$ qui correspondent à quatre critères : le nombre de syllabes (l'échantillon de stimuli comporte 16 mots de 2 syllabes et 24 mots de 3 syllabes), le type d'accentuation (40 stimuli correspondant à trois positions d'accent différentes : 16 mots oxytons, 16 mots paroxytons et 8 mots proparoxytons), la structure accentuelle (combinaison des paramètres 'type d'accentuation' et 'nombre de syllabes') et la transparence lexicale (dans notre expérimentation pour chaque type de mot nous avons sélectionné 4 mots transparents et 4 mots non transparents).

En fonction des variables contrôlées de l'étude, les stimuli ont été sélectionnés selon trois critères. Premièrement, en ce qui concerne la catégorie grammaticale, ces mots appartiennent tous à l'une des deux catégories grammaticales suivantes: substantifs ou adjectifs. Ce choix est guidé par le fait que leur accentuation n'est pas déterminée morphologiquement, comme c'est le cas en espagnol pour les formes verbales (Harris, 1991 : 174). Deuxièmement, concernant la structure syllabique, la syllabe accentuée du mot a une structure CV pour les mots paroxytons et proparoxytons, et une structure CVC pour les mots oxytons. La structure $\mathrm{CV}$ étant la plus courante en général dans les deux langues (Quilis 1981: 335). Troisièmement, quant au nombre de syllabes de la phrase, toutes les phrases possèdent huit syllabes, indépendamment du nombre de syllabes du stimulus concret.

\subsection{Facteurs expérimentaux}

Nous mesurons les résultats obtenus en fonction de huit facteurs :

a) Facteur LM : les résultats obtenus sont analysés en fonction de la langue maternelle du sujet : LM français et LM espagnol.

b) Facteur 'niveau des sujets' : les 32 sujets ont été répartis en 4 groupes et les performances comparées en fonction du niveau de langue des sujets :

- Niveau A : débutants

- Niveau B : intermédiaires

- Niveau C : expérimentés

- Niveau D : hispanophones

c) Facteur 'isolés/mots en contexte' (phrases à intonation descendante ou phrases à intonation montante) : les mots cibles sont présentés dans trois conditions :

- Condition $1:$ mots isolés

- Condition 2 : phrases à intonation descendante

- Condition 3 : phrases à intonation montante

TIPA. Travaux interdisciplinaires sur la parole et le langage, 30 | 2014 
d) Sous-type du 'facteur mots isolés/mots en contexte' (phrases à intonation descendante ou phrases à intonation montante) : facteur 'type d'intonation' pour les phrases

Lorsque le stimulus apparait en contexte, il est toujours situé à la fin de la phrase afin d'étudier l'effet de l'intonation sur la production de l'accent lexical. A ce sujet, trois conditions sont examinées :

- Condition $1:$ mots isolés

- Condition 2 : phrases à intonation descendante

- Condition 3: phrases à intonation montante

e) Facteur 'type d'accentuation': on observe les effets de différents types d'accentuation quant à la production de l'accent lexical espagnol. Dans les stimuli on compte trois types d'accentuation :

- Mots oxytons

- Mots paroxytons

- Mots proparoxytons

f) Facteur 'nombre de syllabes' :

- Mots de 2 syllabes

- Mots de 3 syllabes

g) Facteur 'structure accentuelle' : combinaison des deux facteurs précédents. Cinq structures accentuelles différentes sont considérées:

- Condition $1:$ mots de 2 syllabes oxytons

- Condition 2 : mots de 2 syllabes paroxytons

- Condition 3 : mots de 3 syllabes oxytons

- Condition 4 : mots de 3 syllabes paroxytons :

- Condition 5 : mots de 3 syllabes proparoxytons

\section{h) Facteur 'transparence lexicale'}

- Condition $1:$ mots transparents

- Condition 2 : mots non transparents

Nous analysons 3840 observations : 32 sujets face à 40 stimuli présentés dans 3 conditions.

\subsection{Procédure de passation des tests}

34 L'expérience s'est déroulée dans une salle d'enregistrement. Les sujets ont été placés face à un poste informatique et ont passé l'expérience un par un. Ils effectuent le test de production en prononçant les stimuli à l'aide d'images ${ }^{11}$. Les images sont présentées dans une phrase qu'ils doivent lire. L'image représente un mot. Le sujet ne voit pas le mot qu'il doit prononcer afin d'éviter des effets dus à la lecture ou à la visualisation du mot écrit (comme nous avons dit précédemment, par exemple, la connaissance orthographique du sujet par rapport aux règles d'accentuation). Il prononce le mot qui correspond à l'image. Par exemple, il voit une tomate et prononce le mot « tomate », sans article. Ensuite, il lit la phrase où l'image est insérée et dans laquelle l'image du mot-cible se trouve en position finale, avec l'intonation correspondante ${ }^{12}$. 


\section{¿ Lo cocino}

con ?

Aucune limite de temps n'est imposée aux sujets. Ils passent d'un stimulus au suivant en cliquant avec la souris. Les stimuli apparaissent dans un ordre aléatoire qui est le même pour tous les sujets ${ }^{13}$ grâce au logiciel Présentation, qui permet de faire se succéder les images les unes après les autres au déclenchement d'un clic de souris, l'image suivante s'affichant après un intervalle de temps variant de 1 à 5 secondes, aléatoirement. En même temps les réponses des sujets sont enregistrées à l'aide d'un microphone ${ }^{14}$ et un fichier audio .wav est créé (fréquence d'échantillonnage de $44100 \mathrm{~Hz}$ ).

\subsection{Procédure d'analyse de résultats}

Le logiciel Statistica 8 est utilisé pour l'analyse des résultats des pourcentages de bonnes réponses ou d'erreurs afin de réaliser les tests statistiques pertinents.

\section{Résultats}

\subsection{Effet de la LM}

L'effet de la LM est significatif : $F(1,30)=12,882, p=00116$. En effet, les sujets hispanophones obtiennent $100 \%$ de BR, alors que les francophones atteignent $80,69 \%$ de BR. Nous avons comparé chacun des niveaux aux hispanophones; des différences significatives des niveaux A et B et des hispanophones sont apparues. Toutefois, la production des sujets du niveau $C$ n'est pas significativement différente de celle des sujets natifs $F(3,28)=50,460, p=, 00000$. 


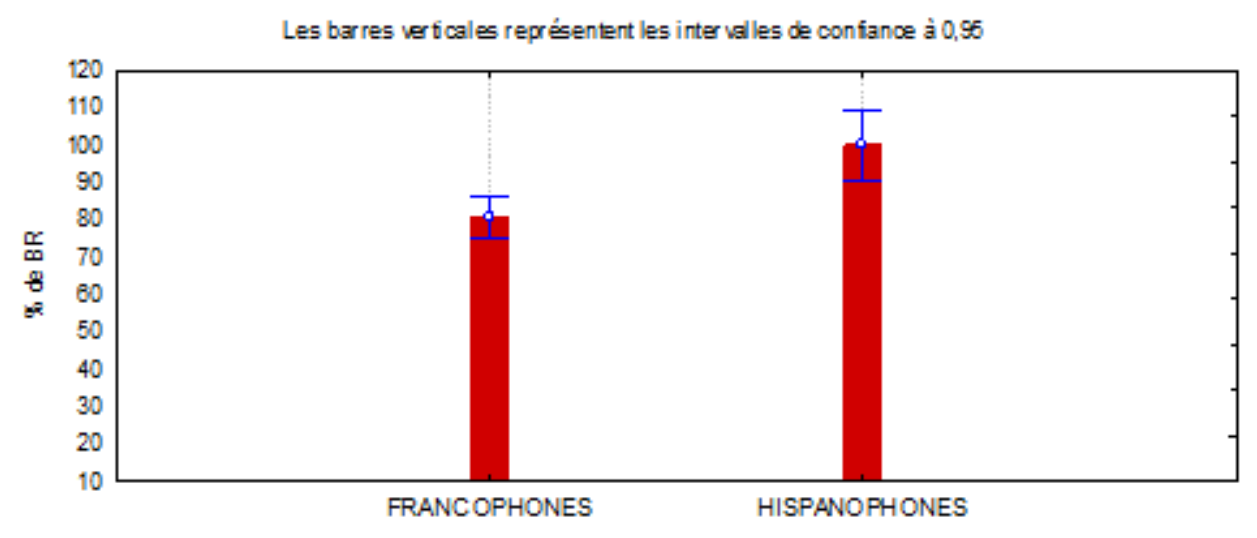

Figure 1 : Pourcentages de bonnes réponses de l'effet du facteur $L M$, tous facteurs confondus

\subsection{Effets globaux}

Nous présentons ci-dessous les résultats restreints aux trois groupes des sujets francophones, les sujets hispanophones ayant obtenu $100 \%$ de bonnes réponses dans toutes les conditions.

\subsubsection{Effet du facteur 'niveau des sujets'}

Nous observons un effet significatif du 'niveau des sujets', toutes conditions confondues : $F(2,21)=36,560, p=, 00000$. Les sujets qui présentent le pourcentage de bonnes réponses le plus faible sont ceux du niveau A, avec $66,97 \%$ de bonnes réponses. Les sujets du niveau B obtiennent un pourcentage plus élevé, $76,97 \%$ de BR. Les sujets du niveau $C$ sont ceux qui ont le taux de réussite le plus élevé : 98,12\% de BR. Le test de Tukey indique que ces différences sont significatives entre les trois niveaux des sujets, c'est-à-dire que les pourcentages de bonnes réponses des sujets du niveau A sont différents de ceux du niveau $B$ et $C$, et les pourcentages de BR sont aussi significativement différents entre les sujets du niveau B et C.

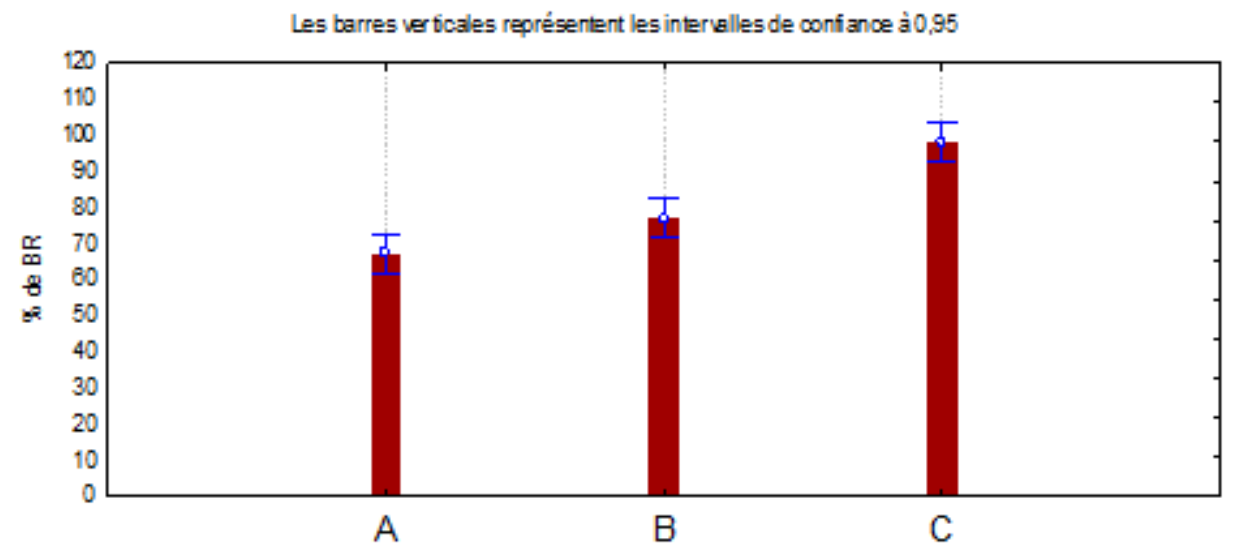

Figure 2 : Pourcentages de bonnes réponses de l'effet du facteur 'niveau des sujets', tous facteurs confondus 


\subsubsection{Effet du facteur 'mots isolés/phrases à intonation descendante et phrases à intonation montante'}

La condition mots à intonation montante (82,70\% de bonnes réponses) obtient le taux de réussite le plus élevé. Dans la condition mots à intonation descendante nous notons $80,52 \%$ de BR contre 78,85\% dans la condition mots isolés $(F(2,42)=3,7661, p=, 03130)$.

Il n'y a donc pas de différences significatives en production entre le traitement de l'accent lexical dans la condition phrases à intonation descendante et la condition phrases à intonation montante (test de Tukey). La réalisation correcte concernant l'accentuation des mots en contexte ne dépend donc pas du type d'intonation.

On n'observe pas de différences significatives entre la condition mots isolés et la condition phrases à intonation descendante pour la production de l'accent lexical. En revanche, l'effet de la production entre la condition mots isolés et la condition phrases à intonation montante est significatif: $F(1,21)=6,4445, p=, 01911$. Les sujets produisent mieux l'accent lexical lorsque les mots sont insérés dans une phrase à intonation montante ( $82,70 \%$ de $\mathrm{BR})$ que lorsqu'ils doivent les prononcer isolément ( $78,85 \%$ de $\mathrm{BR})$.

Nous observons donc seulement des différences significatives entre les mots isolés et les phrases à intonation montante.

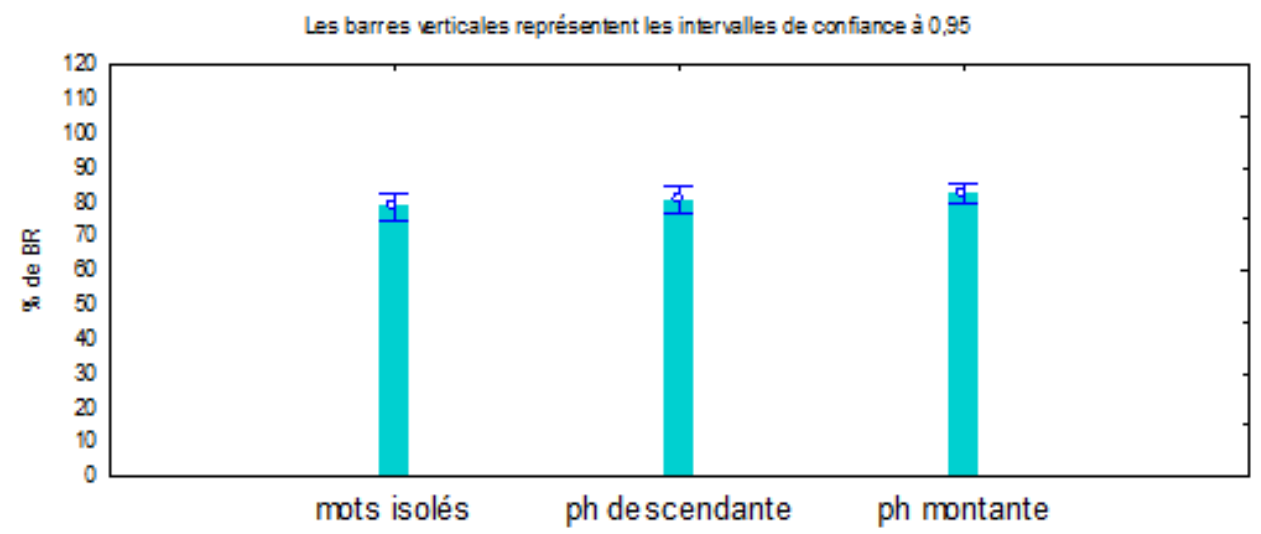

Figure 3 : Pourcentages de bonnes réponses de l'effet du facteur 'mots isolés / phrases à intonation descendante / phrases à intonation montante', tous facteurs confondus

\subsubsection{Effet du facteur 'nombre de syllabes'}

La production de l'accent lexical est plus facile pour les mots de deux syllabes ( $87,32 \%$ de $B R)$ que pour les mots de trois syllabes $(76,27 \%$ de $B R): F(1,21)=23,769, p=, 00008)$. 


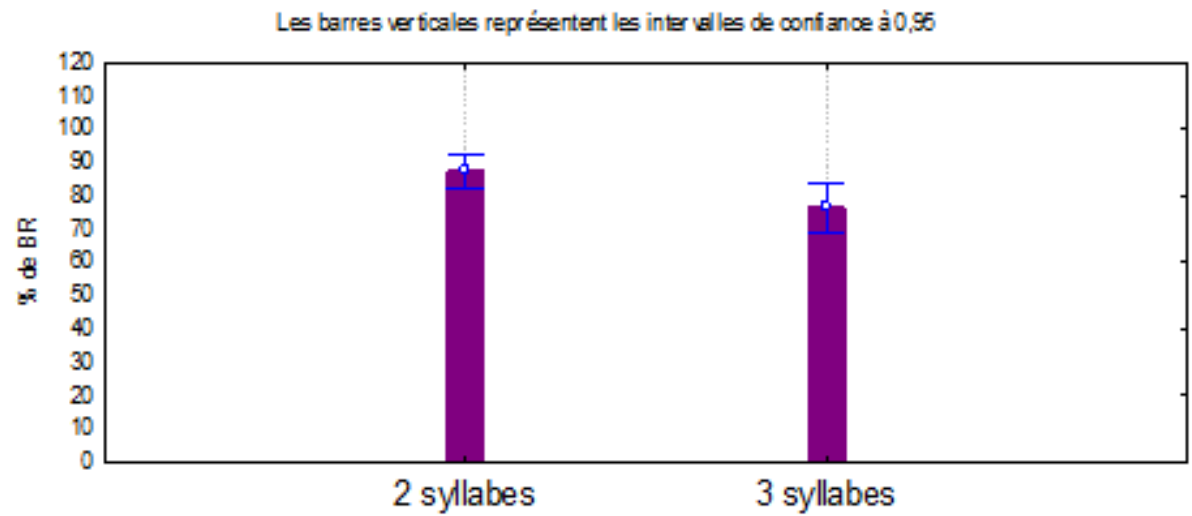

Figure 4 : Pourcentages de bonnes réponses de l'effet du facteur 'nombre de syllabes', tous facteurs confondus

\subsubsection{Effet du facteur 'type d'accentuation'}

Nous observons un effet significatif du facteur 'type d'accentuation': $F(2,42)=16,452$, $\mathrm{p}=, 00001$. En production, le type d'accentuation le plus difficile à traiter est l'accentuation proparoxytone, les sujets ayant obtenu un score $63,36 \%$ de BR, suivie par l'accentuation oxytone (77,34\% de BR). Les meilleures performances sont obtenues pour l'accentuation paroxytone $(92,70 \%$ de BR). Tous les types d'accentuation sont significativement différents entre eux (test de Tukey).

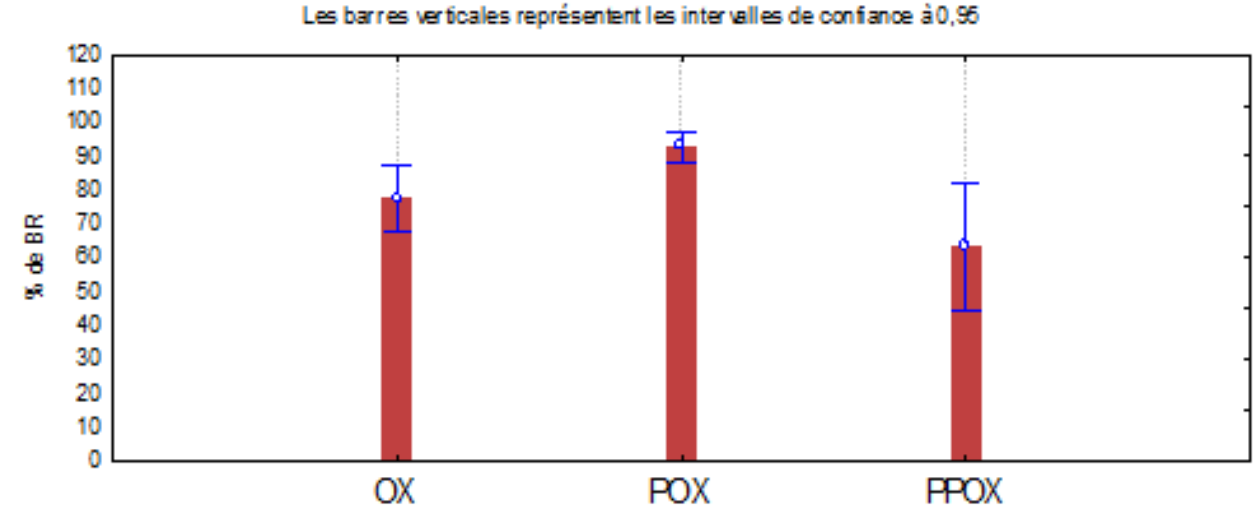

Figure 5 : Pourcentages de bonnes réponses de l'effet du facteur 'type d'accentuation', tous facteurs confondus

\subsubsection{Effet du facteur 'structure accentuelle'}

L'effet de la 'structure accentuelle', toutes conditions confondues, est significatif ( $\mathrm{F}(4$, $84)=14,815, p=00000$ ). Les sujets rencontrent moins de difficultés avec les mots paroxytons de deux et trois syllabes (92,88\% et $92,53 \%)$. Ils obtiennent des pourcentages inférieurs pour les mots oxytons de deux syllabes $(81,77 \%)$ et les mots oxytons de trois syllabes $(72,91 \%)$. La structure accentuelle la plus difficile à traiter est l'accentuation proparoxytone. Le pourcentage de BR descend à moins de $63,36 \%$. 


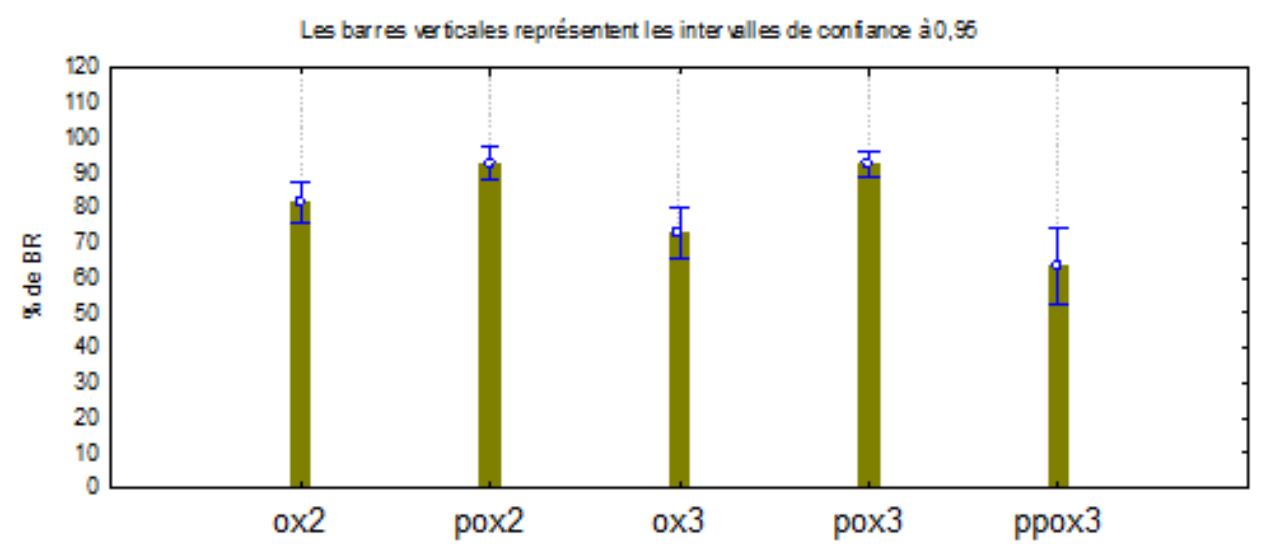

Figure 6 : Pourcentages de bonnes réponses de l'effet du facteur structure accentuelle, tous facteurs confondus

\subsubsection{Effet du facteur 'transparence lexicale'}

L'analyse de variance a permis de mettre en évidence un effet significatif du facteur 'transparence lexicale' : $F(1,21)=12,341, p=, 00207$. En effet, nous avons observé que les sujets obtiennent des scores supérieurs dans la condition mots non transparents $(83,33 \%$ de BR) par rapport à la condition mots transparents (77,98\%). Il semblerait que la similarité phonétique entre les mots en français et les mots en espagnol entraîne des difficultés supplémentaires lors de l'accentuation des mots en production.

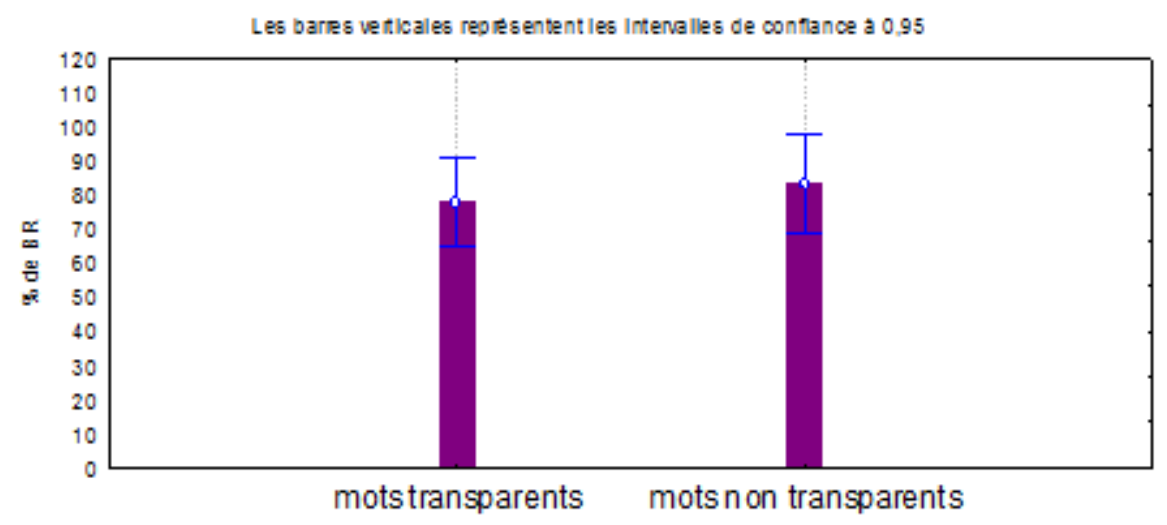

Figure 7 : Pourcentages de bonnes réponses de l'effet du facteur 'transparence lexicale', tous facteurs confondus

\subsubsection{Interaction entre le facteur 'niveau des sujets' et le facteur 'nombre de syllabes'}

Nous avons constaté un effet significatif de l'interaction entre le facteur 'niveau des sujets' et le facteur 'nombre de syllabes' : $F(2,21)=4,9611, p=, 01720$. Le test de Tukey nous permet de noter que les sujets des niveaux A et $B$ ont plus de difficultés en production pour les mots de trois syllabes (60,59 \% et $70,48 \%$ de BR respectivement) que pour les mots de deux syllabes ( $76,56 \%$ et $86,71 \%$ de BR). Les sujets du niveau C traitent les deux conditions de manière équivalente $(97,74 \%$ pour les mots de trois syllabes et $98,69 \%$ pour les mots de deux syllabes). 


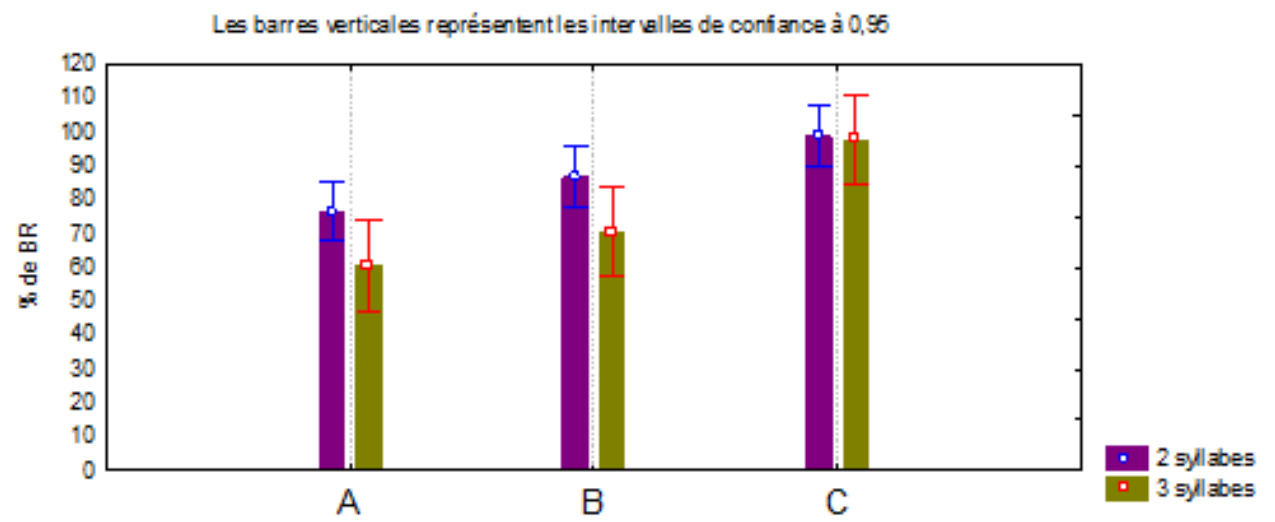

Figure 8 : Pourcentages de bonnes réponses de l'interaction entre le facteur 'niveau des sujets' et le facteur 'nombre de syllabes'

\subsubsection{Interaction entre le facteur 'niveau des sujets' et le facteur 'type d'accentuation'}

Nous avons constaté que l'interaction entre le facteur 'niveau des sujets' et le facteur 'type d'accentuation' est significative: $F(4,42)=4,4986, p=, 00408$. Le test de Tukey précise que les sujets des niveaux A et $B$ traitent différemment les mots paroxytons $(87 \%$ et $92 \%$ de BR respectivement) que les mots proparoxytons (niveau A : $38 \%$ et niveau B : $52 \%$ de $\mathrm{BR}$ ) et les mots oxytons ( $60 \%$ et $74 \%$ de BR respectivement), obtenant des performances beaucoup plus faibles dans ces deux derniers cas. Les sujets du niveau $\mathrm{C}$ traitent les trois types d'accentuation de manière similaire ( $96 \%$ pour les oxytons, $98 \%$ pour les paroxytons et $99 \%$ pour les proparoxytons de BR).

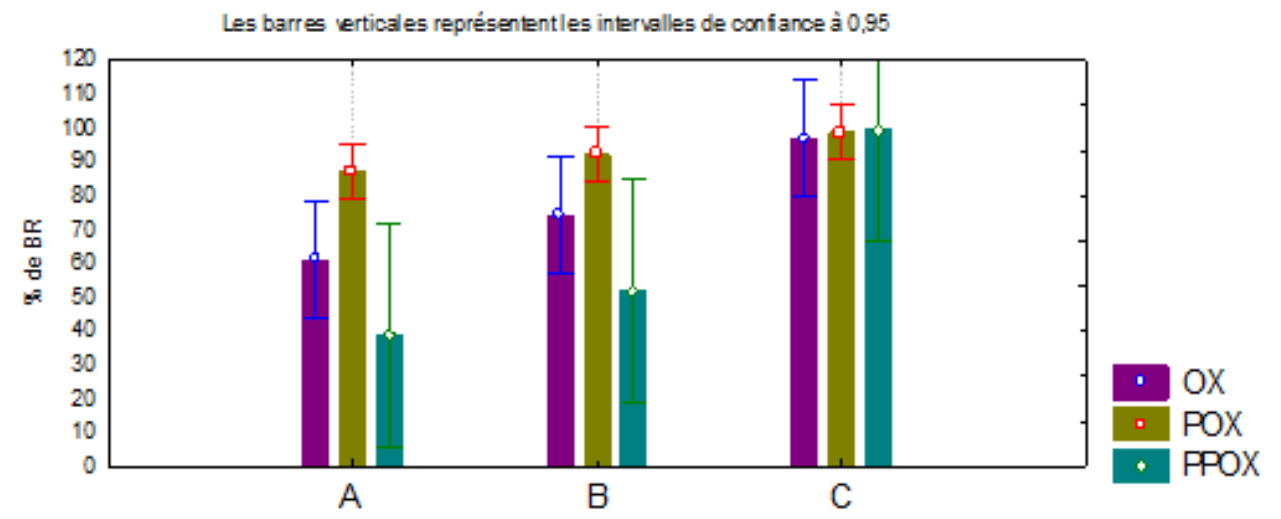

Figure 9 : Pourcentages de bonnes réponses de l'interaction entre le facteur 'niveau des sujets' et le facteur 'type d'accentuation'

\subsubsection{Interaction entre le facteur 'niveau des sujets' et le facteur 'structure accentuelle'}

L'interaction entre le facteur 'niveau des sujets' et le facteur 'structure accentuelle' est significative: $F(8,84)=3,8857, p=, 00061$. Nous observons que les sujets du niveau $C$ obtiennent des résultats homogènes dans toutes les conditions d'accentuation, avec un pourcentage de bonnes réponses aux alentours de $98 \%$. Les sujets des niveaux A et B présentent les mêmes difficultés avec le traitement de l'accent en fonction de la structure 
accentuelle. Nous pouvons observer avec cette analyse que les pourcentages de BR sont plus bas pour les sujets du niveau A. Ainsi, le pourcentage de BR pour les mots paroxytons est environ de $92 \%$ pour les sujets du niveau B et de $87 \%$ pour les sujets du niveau A. Les mots oxytons s'avèrent plus difficiles à prononcer pour les deux niveaux : niveau $\mathrm{B}: 81 \%$ pour les mots de deux syllabes et $67 \%$ de BR pour les mots de trois syllabes, et niveau A : $66 \%$ et $55 \%$ de BR respectivement. La prononciation de l'accent lexical des mots proparoxytons est la plus difficile de toutes les conditions. Les sujets du niveau B obtiennent $52 \%$ de BR tandis que les sujets du niveau A ne parviennent qu'à $38 \%$ de BR.

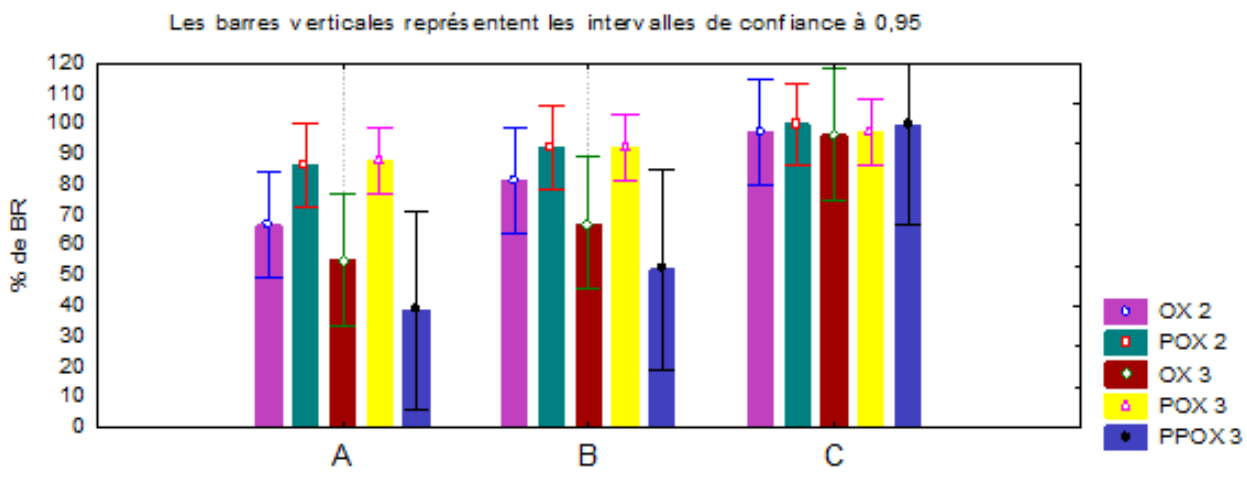

Figure 10 : Pourcentages de bonnes réponses de l'interaction entre le facteur 'niveau des sujets' et le facteur 'structure accentuelle'

\subsubsection{Interaction du facteur 'niveau des sujets' et le facteur 'transparence lexicale'}

Dans l'interaction entre le facteur 'niveau des sujets' et le facteur 'transparence lexicale' nous n'observons pas de différences significatives : $F(2,21)=, 81470, p=, 45628$.

\section{Discussion}

52 Nous avions supposé, de la part du sujet francophone, une tendance à être influencé par sa $\mathrm{LM}$ et nous nous attendions à des productions incorrectes fréquentes au niveau accentuel. Les résultats indiquent que, dans $80 \%$ des cas, les accents ont bien été placés. L'effet de la LM tous niveaux confondus existe, mais il est moins marqué que ce que nous avions envisagé.

53 Nous avons mis en évidence un effet du 'niveau des sujets' dans toutes les conditions. Nous avons observé que les performances au niveau de la production s'améliorent avec l'expertise en langue des sujets. Plus le niveau de langue est élevé, moins on observe d'erreurs en production. L'hypothèse d'un effet du niveau en production est pleinement validée. Par ailleurs, les différences entre les trois groupes de niveau sont globalement significatives. Cet effet a été confirmé dans les trois conditions (mots isolés, phrases à intonation descendante et phrases à intonation montante). Ces résultats vont dans le même sens que ceux rapportés par Lafrance (2001: 111). En revanche, l'auteur observe $75,7 \%$ de BR de la part des sujets experts, que l'auteur dénomme " sujets avancés " ${ }^{15}$. Les sujets qui ont participé à notre expérience ont des performances autour de $98 \%$ de BR, un taux considérablement plus élevé. Nous pouvons supposer que cette meilleure performance est due à un meilleur niveau de langue car, comme nous avons mentionné 
précédemment, pour les sujets experts qui ont participé à notre expérience l'espagnol était une matière principale dans leurs études.

Les différences inter-niveaux suggèrent l'existence de divers stades d'interlangue dans l'acquisition de la prosodie, particulièrement de l'accentuation. Des études réalisées sur l'acquisition d'autres langues ont déjà montré des résultats dans ce sens (Rasier, 2003 ; Wieden, 1993 ; Grosser, 1993), mettant en évidence des caractéristiques de productions en fonction de la LM de l'apprenant et de la langue cible.

Les résultats révèlent qu'il n'y a pas de différences significatives entre la réalisation de l'accent des 'mots isolés' et des 'mots en contexte'. Toutefois, les sujets ont mieux produit l'accent lexical dans la condition phrases à intonation montante que lorsqu'ils prononcent les mots de manière isolée. Il semblerait qu'en production, l'intonation montante puisse se révéler être un élément facilitateur pour la production de l'accent lexical.

56 Les résultats ont mis en exergue un effet du 'nombre de syllabes', ce qui confirme l'hypothèse avancée dans le point 2.2.4. et va dans le même sens que les résultats de Lafrance $^{16}(2001: 112)$. En effet, la réalisation de l'accent est plus facile lorsque les sujets prononcent les mots de deux syllabes que quand ils prononcent ceux de trois syllabes. Un examen attentif de cet effet en fonction du niveau de langue des sujets montre que le nombre de syllabes n'affecte pas la production des sujets experts. Cependant, la production des sujets des niveaux débutants et intermédiaires se voit influencée par le nombre de syllabes du mot. Les performances de BR sont meilleures lors de la production des mots de deux syllabes que de ceux de trois syllabes. Il en est ainsi pour toutes les conditions (mots isolés, phrases à intonation descendante et phrases à intonation montante). Dans ce cas précis, les mots de deux syllabes sont des éléments facilitateurs pour la production de l'accent en espagnol.

Il est apparu que le 'type d'accentuation' joue un rôle lors de la prononciation de l'accent lexical par des francophones. En effet, les sujets éprouvent davantage de difficultés à prononcer l'accent des mots proparoxytons et oxytons que paroxytons. Les différences entre les trois types d'accentuation sont significatives. L'hypothèse de départ est confirmée au niveau global. Il convient de mentionner que, lorsque l'on examine les performances des sujets en fonction du niveau de langue, deux constats s'imposent. Premièrement, comme avec d'autres effets, il n'y a pas de différences de traitement de l'accentuation de la part des sujets experts, qui obtiennent un taux très élevé de bonnes réponses. Deuxièmement, nous observons que pour les sujets des niveaux A et $\mathrm{B}$, il y a de grandes différences entre les performances obtenues pour les mots paroxytons, similaires à celles des sujets experts (pas de différences significatives), ainsi que pour les deux autres types d'accentuation, où les productions correctes des accents lexicaux diminuent considérablement, spécialement pour les mots proparoxytons. Ces différences de résultats en fonction du niveau de langue mettent en lumière que l'acquisition des différents patrons accentuels semble s'effectuer de la manière suivante : en premier lieu le patron paroxyton, dès un niveau débutant. En deuxième lieu le patron oxyton, à partir d'un niveau intermédiaire. Enfin le patron proparoxyton, qui est acquis plus tardivement que les deux autres. De bonnes performances ne sont constatées que lors des productions des sujets experts. L'acquisition des trois patrons accentuels semble être en relation avec la fréquence du patron en espagnol, et par là même à l'exposition de l'apprenant aux différents patrons. Sur un plan didactique, l'enseignant peut en tenir compte pour les programmations ou la création de matériel, en adaptant les difficultés en fonction du 
niveau (insistant, par exemple, sur l'accentuation oxytone et proparoxytone dès les niveaux débutant et intermédiaire).

\section{dans toutes les conditions (mots isolés, phrases à intonation descendante et phrases à} intonation montante). Par ailleurs, le facteur 'structure accentuelle' en interaction avec le 'niveau des sujets' révèle des différences significatives. Globalement, la même constance de résultats des sujets du niveau $C$ s'observe pour toutes les structures accentuelles. En revanche, des résultats similaires de la part des sujets débutants et intermédiaires ne se constatent que pour les mots paroxytons, de deux et de trois syllabes. Pour les autres structures accentuelles les performances se dégradent en fonction du nombre de syllabes pour les oxytons (meilleures productions des mots de deux syllabes que de trois syllabes) et les mots proparoxytons sont toujours les plus difficiles à prononcer pour les sujets des niveaux $\mathrm{A}$ et $\mathrm{B}$. Ces résultats renforcent les conclusions relatives à l'acquisition de différents patrons accentuels.

Les pourcentages ont pu montrer un effet global significatif de 'transparence lexicale'. L'accentuation des mots transparents a été plus difficile que celle des mots non transparents. La transparence lexicale s'avère ne pas être un élément facilitateur, mais, au contraire, le fait que les mots se ressemblent phonétiquement entre les deux langues pose des difficultés supplémentaires au niveau de la réalisation de l'accent. L'hypothèse se voit ici confirmée.

\section{Conclusion}

L'accentuation est un phénomène complexe où de nombreux paramètres interviennent aussi bien au niveau acoustique qu'au niveau phonologique, affectant l'organisation et la compréhension du discours. Cette étude souligne l'intérêt de prendre en compte les caractéristiques de l'interlangue pour mieux comprendre le processus d'acquisition de la prosodie. Les données obtenues montrent que l'apprentissage des éléments prosodiques, notamment l'accentuation, relève d'un processus d'acquisition qui évolue peu à peu et montre des étapes d'interlangue différentes selon le stade d'acquisition phonique de l'apprenant. Nous avons ainsi pu mettre en évidence des contextes facilitateurs pour la production de l'accent lexical. Nous ne prétendons pas, bien entendu, établir un inventaire exhaustif des difficultés que l'on peut rencontrer, mais les résultats obtenus permettent d'avoir une idée plus précise sur des phénomènes de production de l'accentuation.

61 Cette étude centrée sur l'interlangue met en évidence des caractéristiques de productions des apprenants francophones de l'espagnol, mais il serait intéressant d'étendre le protocole d'observation à d'autres langues, ce qui permettrait de questionner de manière expérimentale le processus d'acquisition de l'accent lexical espagnol. Cela permettrait d'identifier ce qui caractérise l'interlangue des apprenants francophones -ou des locuteurs d'autres langues- et ce qui relève plus largement des stratégies d'apprentissage.

D'un point de vue didactique, ce travail a permis de dégager des pistes intéressantes à développer. En effet, grâce à l'étude que nous avons menée et aux données recueillies, nous sommes en mesure d'évaluer d'une façon plus adéquate les besoins des apprenants francophones. Cette étude de l'interlangue au niveau prosodique peut se révéler efficace pour l'enseignement de l'ELE. Elle nous a permis de ressembler des informations

TIPA. Travaux interdisciplinaires sur la parole et le langage, 30 | 2014 
permettant à l'enseignant d'établir une hiérarchie de besoins à partir des bonnes réponses et des erreurs détectées. En prenant en compte cette hiérarchie, les éléments facilitateurs mis en relief et en fonction des effets du 'niveau des sujets' que nous avons mis en évidence, l'enseignant sera plus à même d'élaborer une programmation de contenus adaptés aux besoins spécifiques de chaque niveau. Il serait intéressant de vérifier si ces données se révèlent efficaces d'un point de vue didactique en donnant suite à ce travail avec une expérimentation didactique.

\section{BIBLIOGRAPHIE}

Archibald, J. (1997). The acquisition of second language phrasal stress: a pilot study. In S. J.

Hannahs \& M. Young-Scholten (Eds.), Focus on Phonological Acquisition (pp. 263-289). Amsterdam/ Philadelphia: John Benjamins.

Conseil de l'Europe. (2000). Cadre européen commun de référence pour les langues. Strasbourg: Didier.

Corder, S. P. (1992). A role for the mother tongue. In S. Gass \& L. Selinker (Eds.), Language Transfer in Language Learning. Amsterdam / Philadelphia: John Benjamins Publishing Company.

Cortés Moreno, M. (2002). Didáctica de la prosodia del español: la acentuación y la entonación. Madrid: Edinumen.

Di Cristo, A. (1981). Vers une modélisation de l'accentuation du français. Aix en Provence: Editions de l'Université de Provence.

Fernández González, J., \& de Santiago Guervós, J. (1997). Fonética básica del español para anglohablantes Salamanca: Plaza Universitaria Ediciones.

Gil Fernández, J. (2007). Fonética para profesores de español : de la teoría a la práctica. Madrid: Arco Libros.

Grosser, W. (1993). Aspects of intonation L2 acquisition. In B. Ketterman \& W. Wieden (Eds.), Current Issues in European Second Language Acquisition Research (pp. 81-94). Tübingen: Gunter Narr Verlag.

Harris, J. W. (1991). La estructura silábica y el acento en español. Madrid: Visor Distribuciones.

Iruela, A. (2004). Adquisición y enseñanza de la pronunciación en lenguas extranjeras. Thèse de doctorat, Universidad de Barcelona, $385 \mathrm{p}$.

Lafrance, J. (2001). La production de l'accent espagnol par des francophones. Colloque des Etudiants en Sciences du Langage, Montréal, p. 105-118

Léon, P. (1992). Phonétisme et prononciations du français. Paris: Nathan.

Quilis, A. (1981). Fonética acústica de la lengua española Madrid: Gredos (Biblioteca Románica Hispánica, Manuales, 49).

Quilis, A. (1993). Tratado de fonología y fonética españolas. Madrid: Gredos (Biblioteca Románica Hispánica, Manuales, 74). 
Muñoz García, M., Panissal, N., Billières, M., \& Baqué, L. (2009). La metáfora de la criba fonológica se puede aplicar a la percepción del acento léxico español? Estudio experimental con estudiantes francófonos. In C. M. e. a. Bretones (Ed.), La lingüística aplicada actual: Comprendiendo el lenguaje y la mente (pp. 489-499). Almería: Universidad de Almería AESLA.

Muñoz García, M. (2010) La perception et la production de l'accent lexical de l'espagnol par des francophones: aspects phonétiques et psycholinguistiques, Thèse de doctorat, Université de Toulouse, $576 \mathrm{p}$.

Rasier, L. (2003). Le système accentuel de l'interlangue d'apprenants francophones du néerlandais. Interfaces Prosodiques, Nantes, p. 79-84.

Rasier, L., \& Hiligsmann, P. (2007). Prosodic transfert from L1 to L2. Theoretical and Methodological issues. Nouveaux cahiers de linguistique française, 28 (2007), 41-66.

Rossi, M. (1999). L'intonation du français: description et modélisation. Paris: Ophrys.

Sarmiento, J. A., Jiménez, P., \& Koberski, E. (1974). L'application de l'appareil SUVA-GLINGUA de correction phonétique à l'enseignement de l'espagnol aux francophones. Revue de Langues Vivantes, 40(7), 671-695.

Selinker, L. (1972). La interlengua. In J. Muñoz Liceras (Ed.), La adquisición de las lenguas extranjeras (pp. 79-101). Madrid: Visor.

Vázquez, G. (1992). ¿Errores? ¡Sin falta! Madrid: Edelsa.

Wieden, W. (1993). Aspects of acquisitional stages. In B. Ketterman \& W. Wieden (Eds.), Current Issues in European Second Language Acquisition Research (pp. 125-135). Tübingen: Gunter Narr Verlag.

\section{ANNEXES}

\section{Liste de stimuli des mots isolés}

\begin{tabular}{|l|l|l|l|l|l|}
\hline & OX2 & POX2 & OX3 & POX3 & PPOX3 \\
\hline NON TRANSPARENTS & MUJER & LECHE & VEINTIDOS & CABEZA & DECIMO \\
& PINTOR & CAMA & TENEDOR & ZAPATO & MEDICO \\
& PARED & GATO & CALCETIN & CAMISA & PAJARO \\
\hline TRANSPARENTS & MRANCES & MOTO & ASCENSOR & ESPAÑA & PUBLICO \\
& MARRON & TAXI & MARATON & GUITARRA & MUSICA \\
& MELON & DUCHA & HOSPITAL & TOMATE & CAMARA \\
& PARIS & ROSA & JAPONES & BOTELLA & NUMERO \\
\hline
\end{tabular}


2. Ordre de passation des stimuli en contexte 


\begin{tabular}{|c|c|}
\hline ¿Tú te pones el calcetín? & ¿Ellos corren un maratón? \\
\hline Pablo duerme en su cama & ¿Lo ponemos en la mesa? \\
\hline ¿Lo filmo con la cámara? & ¿Tiene dolor de cabeza? \\
\hline ¿En verano como melón? & ¿Nos ponemos la camisa? \\
\hline ¿Buscamos este zapato? & ¿Bebes zumo de manzana? \\
\hline Me lo dice de corazón & Lo filmo con la cámara \\
\hline ¿Hay vino en la botella? & ¿En Francia se habla francés? \\
\hline Comes carne con tenedor & ¿El señor Cruz es médico? \\
\hline Aquí cogen el número & Esta capital es París \\
\hline ¿David escucha música? & David escucha música \\
\hline ¿Esta capital es París? & Te lavas en una ducha \\
\hline ¿Van de viaje a España? & Alfonso bebe la leche \\
\hline Nos ponemos la camisa & ¿Teresa es una mujer? \\
\hline ¿José toca la guitarra? & Antonio pinta la pared \\
\hline ¿Antonio pinta la pared? & ¿En Japón hablan japonés? \\
\hline ¿Este vestido es marrón? & En verano como melón \\
\hline ¿Lo cocino con tomate? & ¿Mi piso es el décimo? \\
\hline ¿No trabajas el sábado? & ¿Subimos con el ascensor? \\
\hline Alejandro es un pintor & En Francia se habla francés \\
\hline Lo ponemos en la mesa & Vamos a coger un taxi \\
\hline El loro es un pájaro & ¿Me lo dice de corazón? \\
\hline Ellos corren un maratón & ¿Aquí cogen el número? \\
\hline ¿El loro es un pájaro? & ¿Comes carne con tenedor? \\
\hline El señor Cruz es médico & Teresa es una mujer \\
\hline ¿No te gusta este gato? & Aquel pantalón es rosa \\
\hline ¿Alfonso bebe la leche? & Van de viaje a España \\
\hline No trabajas el sábado & Este vestido es marrón \\
\hline ¿Quiere conducir la moto? & ¿Vamos a coger un taxi? \\
\hline Trabajan en un hospital & No te gusta este gato \\
\hline Tiene dolor de cabeza & Subimos con el ascensor \\
\hline José toca la guitarra & ¿Trabajan en un hospital? \\
\hline Hay vino en la botella & ¿Pablo duerme en su cama? \\
\hline ¿El mar es de color azul? & ¿Alejandro es un pintor? \\
\hline En clase somos veintidós & Bebes zumo de manzana \\
\hline El mar es de color azul & En Japón hablan japonés \\
\hline Cantó para su público & Quiere conducir la moto \\
\hline $\begin{array}{l}\text { ¿En clase somos veintidós? } \\
\text { TIPA. Travaux interdisciplinaires su }\end{array}$ & $\begin{array}{l}\text { Tú te pones el calcetín } \\
\text { la parole et le langage, } 30 \text { | } 2014\end{array}$ \\
\hline Mi piso es el décimo & Lo cocino con tomate \\
\hline ¿Aquel pantalón es rosa? & Buscamos este zapato \\
\hline ¿Te lavas en una ducha? & ¿Cantó para su público? \\
\hline
\end{tabular}




\section{NOTES}

1. Le terme d'interlangue a été avancé par Selinker (1972) pour faire référence à un système linguistique structuré et organisé, propre à une étape de l'apprentissage d'une LE. Corder (1992: 21) la définit comme un système intermédiaire entre la LM et la langue cible, d'où le terme interlangue: "The systematic knowledge of language which is independent of both the learner's L1 and the L2 system he is trying to learn ». Nous entendons par interlangue donc un système transitoire qui reflète les connaissances linguistiques d'un individu à un moment donné de l'apprentissage. Ce système se caractérise par la présence ou l'absence de règles qui indiquent le degré de proximité par rapport à la compétence native (Vázquez, 1992 : 133).

2. Traduction : el límite (substantif masculin) : la limite ; limite : limitez (impératif) ou que je limite ou qu'il limite ou que vous limitiez (subjonctif); limité : je limitai (passé simple).

3. On pourrait se demander si les apprenants passent par une représentation graphique du mot même s'ils ne le voient pas à l'écrit. Cette question est légitime mais nous n'avons pas pris en considération cette problématique. Il est possible que les étudiants experts ou ceux du niveau intermédiaire se "représentent " le mot avant de le prononcer et que cette représentation prenne en compte les règles orthographiques d'accentuation, ce qui pourrait avoir une influence sur leur façon de prononcer et accentuer le mot. Cependant, nous ne pensons pas que les apprenants d'un niveau élémentaire (ayant eu entre 60-120 heures de cours) soient influencés par l'orthographe à ce stade de leur apprentissage. En outre, il serait intéressant de voir à quel niveau de leur apprentissage, et donc à quel stade de l'interlangue se produit cette assimilation des règles orthographiques de l'accentuation et si cela a une influence ou non dans la manière de prononcer le mot. Nous pourrions poser l'hypothèse d'une acquisition par étapes de l'orthographe où l'écriture de l'accent viendrait en dernier, s'associant alors à une prise en compte de sa valeur phonologique.

4. Nous avons traité les productions du groupe contrôle de sujets hispanophones lorsque nous avons analysé acoustiquement les réalisations acoustiques des sujets francophones et des hispanophones. En ce qui concerne cette expérience, le sujet hispanophone ne se trompe pas dans l'accentuation du mot. Nous n'utilisons que le pourcentage de bonnes réponses des hispanophones afin de vérifier si le résultat des productions globales des sujets francophones est significatif.

5. Étudiantes en espagnol de l'Université Toulouse II.

6. Ce groupe est composé d'apprenants débutants ou faux débutants ayant effectué entre 60 et 120 heures de cours d'espagnol.

7. Il s'agit d'apprenants intermédiaires qui ont suivi un minimum de 180 heures de cours de langue espagnole.

8. Ces sujets possèdent un niveau de langue très élevé. Contrairement aux sujets du niveau A et $B$, les sujets du niveau $C$ ont fait des études de spécialité en langue espagnole pendant plusieurs années et ont souvent séjourné dans un pays de langue espagnole. Tous ces sujets avaient effectué au minimum quatre ans d'études universitaires (niveau de Master 1) dans une discipline ayant comme matière principale l'espagnol, soit en L.L.C.E. soit en L.E.A.

9. Avant de les soumettre à l'expérimentation, nous avons réalisé un entretien oral avec chacun des sujets, afin de vérifier que leur niveau de langue correspondait au niveau requis par l'expérience. Le but étant d'assurer le plus possible l'homogénéité du niveau et garantir le niveau 
réel du sujet. En outre, tous les sujets francophones ne connaissent aucune autre langue romane, dans l'optique d'écarter des interférences, notamment pour la production d'items à l'oral.

10. Ne présentant donc a priori aucune difficulté majeure même pour les sujets du niveau A. Les mots étaient connus pour tous les locuteurs puisque, comme nous le détaillons dans le point 3.3., le sujet ne voit à aucun moment le mot écrit. Nous n'avons retenu que les productions orales des sujets qui ont prononcé tous les mots.

11. Les images sont issues du programme ClipArt

12. Le fait de prononcer le mot isolé juste avant de le dire en contexte est un choix de protocole afin de limiter le temps de l'expérience et le nombre total d'images. En réalité les sujets ont prononcé 93 stimuli, mais nous n'avons sélectionné que les 40 prononcés par ces 32 sujets.

13. Annexe 2 : ordre de passation des stimuli en contexte en production

14. Référence : BST-Modèle: MC-504-Emetteur : BP-193-UHF Wireless System ; Récepteur : BST-Modèle : HF-Récepteur : UDR-193 ; Carte son : MOTU-Modèle : 896 HD.

15. Les sujets soumis à cette expérience sont, selon l'auteur, tous locuteurs natifs du français repartis selon les trois niveaux d'apprentissage de l'Ecole de langue de l'Université de Québec à Montréal : débutant, intermédiaire et avancé.

16. "Les sujets ont mieux réussi à produire correctement les mots de deux syllabes où ce pourcentage de réussite s'élève à $82,2 \%$. Puis les mots de trois syllabes ont été accentués correctement à $67,9 \%$ ».

\section{RÉSUMÉS}

Située dans le domaine de l'apprentissage des langues étrangères, cette recherche a pour objet l'étude de la production de l'accent lexical espagnol par des francophones. Les systèmes accentuels des deux langues sont différents. Le français est une langue à accent fixe, tandis que l'espagnol est une langue à accent libre avec plusieurs types d'accentuation possibles. Prenant en compte ces éléments, une approche expérimentale a été adoptée afin de tester l'influence de la langue maternelle des francophones sur la production de l'accent lexical espagnol. Les sujets effectuent un test de production en prononçant les stimuli qui sont présentés à la fin d'une phrase. Les résultats révèlent un effet de la langue maternelle tous niveaux de langue confondus. Des différences inter-niveaux ont été mises en évidence suggérant l'existence de divers stades d'interlangue dans l'acquisition de la prosodie, particulièrement de l'accentuation. L'acquisition des différents patrons accentuels semble être la suivante : les francophones acquièrent d'abord l'accentuation paroxytone, ensuite l'oxytone et finalement la proparoxytone. Enfin, les résultats obtenus ont apporté des précisions sur divers aspects de l'acquisition de l'accentuation en espagnol, ce qui d'un point de vue didactique peut servir à proposer un matériel mieux adapté aux besoins spécifiques d'un public francophone.

This paper focuses on second language acquisition. I am particularly interested in the way French learners acquire Spanish prosody and more precisely lexical stress.

French and Spanish display major differences when it comes to lexical stress. French is said to be a fixed-stress language with primary stress systematically falling on the last syllable of phonological phrases (Di Cristo, 1981) whereas Spanish is considered as a free-stress language with stress potentially falling on any of the last four syllables of a word (Quilis, 1993). It has been shown that the mother tongue of a learner strongly influences the production of segmental and 
suprasegmental components in the targeted foreign language (Rasier \& Hiligsmann, 2007; Wieden, 1993). Various researchers observed and documented a strong tendency for French learners to overuse oxytonic stress in Spanish (Iruela, 2004; Gil, 2007). Thus, taking into account the specific prosodic characteristics of both languages, I developed an experimental procedure so as to test and evaluate the influence of the mother tongue on the production of Spanish lexical stress by French learners.

The sample consisted of the recordings of 24 French speakers and 8 Spanish speakers representing the control group. I analysed 3840 productions which correspond to the recordings of 32 different subjects confronted with 40 stimuli under three specific conditions. The subjects carried out a production test that consisted in pronouncing the stimuli triggered by pictures that were introduced at the end of a sentence they had to read. Each picture represents a word. The subjects do not see the words that they are asked to pronounce in order to avoid any interference that might result from reading the written words. First, words are pronounced in isolation, and then they are inserted at the end of a sentence and pronounced in context (in connected speech). For instance, when the subjects are exposed to the picture of a guitar, they pronounce the word " guitarra " in isolation, without using any article. Then they read the sentence in which the picture was inserted, the target words being placed at the end of each of those sentences and with the appropriate intonation pattern. (For instance: ¿José toca la guitarra?). A single sentence is presented twice, first as a statement (with a falling intonation contour) and then as a question (with a rising intonation contour).

I put forward 8 hypotheses:

1) General hypothesis: The Mother Tongue Effect: I assume that the productions of French learners will be influenced by their mother tongue and that lexical stress will shift to the last syllable.

2) The Proficiency Hypothesis: I assume that most proficient learners will make fewer errors. The subjects are split into three groups depending on their linguistic competence and according to the CEFRL (Common European Framework for Languages, A, B and C).

3) Words in isolation vs. words in context: I suppose that the production of lexical stress will be more challenging for speakers in a sentence than in isolation.

4) The impact of intonation patterns: I assume that the rising intonation contour plays an important part in the accurate production of lexical stress: it might be made more difficult for learners to produce lexical stress with a rising contour than with a falling one.

5) Does the number of syllables have an impact? The longer the word, the more difficult it might be for speakers to assign stress. The stimuli contain two or three syllables (i.e. «a-zul » or « cami-sa »).

6) What is the effect of the position of stress? Two hypotheses are put forward here: first, I assume that the speakers' productions will be better in paroxytonic words (words stressed on the penultimate syllable as in " moto ») since these words are very frequently used in Spanish. Second, I assume that stress positions that are similar in both languages (i.e. oxytonic stress in " fran-"cès ") can be the cause of more difficulty.

7) What is the effect of stress patterns? This factor is a combination of both the impact of the number of syllables and that of stress position. I took into account this factor to check if both hypotheses lead to different effects on learners' productions.

8) What is the effect of lexical transparency? I assume that the effect of phonetic similarity between Spanish and French words will be significant. Indeed, segmental similarities between French and Spanish words can be a source of difficulty. It is expected that the production of lexical stress will be more challenging for «transparent words » (i.e. words that are phonetically close such as «me'lón / melon », «'taxi / taxi ») than for « opaque words » (with no phonetic similarities such as « a'zul / bleu », « re'loj / montre », « 'pájaro / oiseau »). 
The results confirmed the effect of the proficiency factor on the production of lexical stress and revealed the existence of several stages in the development of the learners' interlanguage and in their acquisition of prosody. The acquisition of stress patterns seems to be gradual: first learners acquire paroxytonic stress, then they acquire oxytonic stress and finally they acquire proparoxytonic stress. Stress position seems to have a strong effect on the productions of lexical stress. The best productions were observed in paroxytonic words. This study highlights the existence of facilitating contexts for the acquisition of stress patterns. The students' productions were better in interrogative sentences marked by a rising intonation contour than in isolated words. Furthermore, similarities between the languages in transparent words and oxytonic words were linked to weaker performances.

INDEX

Mots-clés : production, accent lexical, espagnol, français, apprentissage de langues étrangères

Keywords : lexical stress, Spanish, French, Second Language Acquisition

\section{AUTEUR}

\section{MARGARITA MUÑOZ GARCIA}

LIDILE EA 3874, Université de Rennes 2

margarita.munoz-garcia@uhb.fr 